\title{
PERIODS FOR HOLOMORPHIC MAPS VIA LEFSCHETZ NUMBERS
}

\author{
JAUME LLIBRE AND MICHAEL TODD
}

Received 27 June 2004

We characterise the set of fixed points of a class of holomorphic maps on complex manifolds with a prescribed homology. Our main tool is the Lefschetz number and the action of maps on the first homology group.

\section{Introduction and main result}

In this note we are concerned with fixed point theory for holomorphic self maps on complex manifolds. After the well-known Schwarz lemma on the unit disk, which assumes a fixed point, the Pick theorem was proved in [8]. This can be extended to a Pick-type theorem on hyperbolic Riemann surfaces as is shown in $[5,7]$. For a more general type of space: open, connected and bounded subsets of a Banach space, the Earle-Hamilton theorem was proved in [2].

We prove a similar theorem for periodic points of holomorphic maps of complex manifolds with a prescribed homology. Our proof comes from a topological viewpoint and bears no relation with other methods of proof for this problem. We use a result of Fagella and Llibre in [3] which relates the Lefschetz number to the set of fixed points for holomorphic maps on compact complex manifolds. In fact, we show that for many situations, holomorphic maps can have at most one fixed point.

We recall the classical results first. First we state a Pick-type theorem. This is very close to the well-known Pick theorem on the unit disk $\mathbb{D}$, which is in turn very similar to the classical Schwarz lemma on $\mathbb{D}$ (see [7] for both results). Let $\operatorname{Per}(f)$ denote the set of periods of the periodic points of $f$. Also define $\operatorname{Fix}(f)$ to be the set of fixed points of $f$ and define $\operatorname{Per}_{m}(f)$ to be the set of periodic points of period $m$.

The following Pick-type theorem is [7, Theorem 5.2].

Theorem 1.1. Suppose that $S$ is a hyperbolic Riemann surface. Then for a holomorphic map $f: S \rightarrow S$,
(a) either $f^{p}$ is the identity for some $p$;
(b) or $f$ has one periodic point: a fixed point;
(c) or $f$ no periodic points. 
The following is the Earle-Hamilton theorem, see [2,4] for more details. (In fact, the usual setting for this theorem is complex Banach spaces.) We say that a set $E$ is mapped strictly inside a subset $F$ of the metric space $X$ by the map $f$ if there is some $\epsilon>0$ such that $B_{\epsilon}(f(E)) \subset F$ where $B_{\epsilon}(A)$ is the $\epsilon$-neighbourhood of the set $A \subset X$.

Theorem 1.2. Let $M \subset \mathbb{C}^{m}$ be a bounded connected open set and $f: M \rightarrow M$ be holomorphic. If $f(M)$ lies strictly inside $M$ then $f$ has a unique fixed point.

Note that if the map $f$ satisfies the theorem then so does $f^{m}$. Therefore, $\operatorname{Per}(f)=\{1\}$.

Given a complex manifold $M \subset \mathbb{C}^{m}$, let $f: M \rightarrow M$ be a holomorphic map which extends continuously to $\bar{M}$ the closure of $M$, and furthermore, so that $f(\bar{M})$ is inside $\stackrel{\circ}{M}$, the interior of $M$. Here we take the closure with respect to the usual topology on $\mathbb{C}^{m}$ and the interior with respect to the topology of $M$. We denote the set of such maps by $\mathscr{H}(M)$. Observe that if $M$ has no boundary then whenever $f: M \rightarrow M$ is holomorphic, we have $f \in \mathscr{H}(M)$. Also note that whenever $M$ is open and bounded then $f \in \mathscr{H}(M)$ maps $M$ strictly inside itself. The following is our main result.

Theorem 1.3. Suppose that $M$ is a complex manifold and $f \in \mathcal{H}(M)$. Suppose further that $H_{1}(M, \mathbb{Q})=\overbrace{\mathbb{Q} \oplus \cdots \oplus \mathbb{Q}}^{n}$ for $n \geq 0$ (when $n=0$ we suppose $H_{1}(M, \mathbb{Q})=\{0\}$ ) and $H_{k}(M, \mathbb{Q})=0$ for $k>1$. Then

(a) either there is some $m \geq 1$ such that $f^{m}$ has infinitely many fixed points;

(b) or $f$ has only one periodic point: a fixed point;

(c) or $f$ has no periodic points.

Furthermore, if $H_{k}(M, \mathbb{Q})=0$ for $k>0$ then we are in case $(b)$.

For example, in case (a) we could consider a rational rotation of the torus; in case (b) we could consider the map $z \mapsto z / 2$ on the unit disk; in case (c) we could consider an irrational rotation of the torus.

Note that our theorem can apply in any dimension and does not require manifolds to be open or bounded. Therefore, in some cases it is an extension of Theorems 1.1 and 1.2.

\section{Proof of the main result}

To prove Theorem 1.3 we use Lefshetz number and Theorem 2.1 below. We recall the concept of Lefschetz number. Let $M$ be a compact manifold of dimension $M$. Then a continuous map $f: M \rightarrow M$ induces an endomorphism $f_{* k}: H_{k}(M, \mathbb{Q}) \rightarrow H_{k}(M, \mathbb{Q})$ for $k=0,1, \ldots, n$ on the rational homology of $M$. The Lefschetz number of $f$ is defined by

$$
L(f)=\sum_{k=0}^{n}(-1)^{k} \operatorname{trace}\left(f_{* k}\right) .
$$

Since $f_{* k}$ are integral matrices, it follows that $L(f)$ is an integer.

By the well-known Lefschetz Fixed Point theorem, if $L(f) \neq 0$ then $f$ has a fixed point (see, for instance, [1]). We can consider $L\left(f^{m}\right)$ too: also $L\left(f^{m}\right) \neq 0$ implies that $f^{m}$ has a fixed point. 
Our main tool here is the following theorem of [3].

Theorem 2.1. Let $M$ be a complex manifold and $f \in \mathscr{H}(M)$ be a nonconstant map. Suppose that for $m \geq 1$, all fixed points of $f^{m}$ are isolated. Then $L\left(f^{m}\right) \geq \# \operatorname{Fix}\left(f^{m}\right)$.

In fact, in [3] the assumption on the periodic points was that the set of all periodic points must be isolated. It is straightforward to weaken this assumption as above. Also, it was assumed that $f$ was holomorphic on a compact manifold $M$ and that $f(M) \subset \stackrel{\circ}{M}$. However, we do not need holomorphicity on the boundary of our manifold since we cannot have any fixed points there.

Remark 2.2. In fact, it is easy to see from the proof of this theorem, that we come to the same conclusions for maps which are not holomorphic, but for which $\operatorname{det}\left(I-D f^{m}(x)\right)>$ 0 for any $x \in \operatorname{Fix}\left(f^{m}\right)$.

We will need the following lemma, see the appendix of [6].

Lemma 2.3. Let $\mu_{1}, \ldots, \mu_{q}$ be complex numbers with $\left|\mu_{j}\right|=1$. For any $\epsilon>0$ there exist infinitely many values of $m$ such that $\left|\mu_{j}^{m}-1\right|<\epsilon$ for all $1 \leq j \leq q$. Hence, $\operatorname{Re}\left(\mu_{j}^{m}\right)>1-\epsilon$ for every $\mu_{j}$.

Proof of Theorem 1.3. We suppose that there is no $m \geq 1$ such that $f^{m}$ has infinitely many fixed points. Then every fixed point for $f^{m}$ is isolated for any $m$. So we have the result of Theorem 2.1. In particular, this means that $L\left(f^{m}\right) \in \mathbb{N} \cup\{0\}$ for all $m \in \mathbb{N}$.

In the case that $H_{k}(M)=0$ for $k>0, f_{* 0}=(1)$, multiplication by 1 ; and for $k>0, f_{* k}$ is the zero map. So we have $L\left(f^{m}\right)=1$ for all $m \geq 1$. Therefore, by the Lefschetz Fixed Point theorem, $f$ has a fixed point. Furthermore, by Theorem 2.1, \# $\operatorname{Fix}\left(f^{m}\right) \leq 1$ for all $m \geq 1$. Therefore, $\operatorname{Per}(f)=\{1\}$.

When $n \geq 1, f_{* 1}$ is an $n \times n$ matrix with integer entries and with eigenvalues $\lambda_{1}, \ldots, \lambda_{n}$, see [9]. So trace $\left(f_{* 1}\right)=\lambda_{1}+\cdots+\lambda_{n}$ and $L\left(f^{m}\right)=1-\lambda_{1}^{m}-\cdots-\lambda_{n}^{m}$.

We reorder the eigenvalues in order of decreasing modulus (when two eigenvalues have the same modulus, any choice of order suffices).

Case 1. Suppose that $\left|\lambda_{1}\right|>1$.

Suppose that $\left|\lambda_{1}\right|=\cdots=\left|\lambda_{k}\right|$ for some maximal $1 \leq k<n$ (the case $k=n$ follows similarly). Consider $\mu_{j}=\lambda_{j} /\left|\lambda_{1}\right|$ for $1 \leq j \leq k$. If we let $\epsilon>0$ as in Lemma 2.3 we have some sequence $m_{l} \rightarrow \infty$ such that $\operatorname{Re}\left(\mu_{1}^{m_{l}}+\cdots+\mu_{k}^{m_{l}}\right)>k(1-\epsilon)$. Therefore,

$$
\operatorname{Re}\left(\lambda_{1}^{m_{l}}+\cdots+\lambda_{k}^{m_{l}}\right)>\left|\lambda_{1}\right|^{m_{l}} k(1-\epsilon), \quad\left|\operatorname{Re}\left(\lambda_{k+1}^{m_{l}}+\cdots+\lambda_{n}^{m_{l}}\right)\right| \leq(n-k)\left|\lambda_{k+1}\right|^{m_{l}} .
$$

Since $\left|\lambda_{1}\right|>\left|\lambda_{k+1}\right|$ there must exist some large enough $m_{l}$ such that

$$
\operatorname{Re}\left(\lambda_{1}^{m_{l}}+\cdots+\lambda_{k}^{m_{l}}\right)>1+\left|\operatorname{Re}\left(\lambda_{k+1}^{m_{l}}+\cdots+\lambda_{n}^{m_{l}}\right)\right| .
$$

Thus $1-\left(\lambda_{1}^{m_{l}}+\cdots+\lambda_{n}^{m_{l}}\right)$ is negative, which is not possible by Theorem 2.1 . 
Case 2. Suppose that $0<\left|\lambda_{1}\right|<1$.

If $\lambda_{2}, \ldots, \lambda_{n}=0$ then $L(f)$ cannot be an integer so this is not possible by the definition of Lefschetz number. If $\left|\lambda_{2}\right|>0$ we will again show that there exists some $m \geq 1$ such that $L\left(f^{m}\right)$ is not an integer. We suppose that $\left|\lambda_{1}\right|=\cdots=\left|\lambda_{k}\right|$ for some maximal $1 \leq k<n$ (the case $k=n$ follows similarly). As in Case 1, we can choose $\epsilon>0$ and let $\mu_{j}=\lambda_{j} /\left|\lambda_{1}\right|$ for $1 \leq j \leq k$. From Lemma 2.3, we have a sequence $m_{l} \rightarrow \infty$ such that $\operatorname{Re}\left(\mu_{1}^{m_{l}}+\cdots+\mu_{k}^{m_{l}}\right)>$ $k(1-\epsilon)$ and so

$$
\operatorname{Re}\left(\lambda_{1}^{m_{l}}+\cdots+\lambda_{k}^{m_{l}}\right)>\left|\lambda_{1}\right|^{m_{l}} k(1-\epsilon), \quad\left|\operatorname{Re}\left(\lambda_{k+1}^{m_{l}}+\cdots+\lambda_{n}^{m_{l}}\right)\right| \leq(n-k)\left|\lambda_{k+1}\right|^{m_{l}} .
$$

Since $\left|\lambda_{1}\right|>\left|\lambda_{k+1}\right|$ there must exist some large enough $m_{l}$ such that

$$
\left|\operatorname{Re}\left(\lambda_{1}^{m_{l}}+\cdots+\lambda_{k}^{m_{l}}\right)\right|>\left|\operatorname{Re}\left(\lambda_{k+1}^{m_{l}}+\cdots+\lambda_{n}^{m_{l}}\right)\right|
$$

so $\operatorname{Re}\left(\lambda_{1}^{m_{l}}+\cdots+\lambda_{k}^{m_{l}}\right) \neq 0$. Since for large $m_{l}$, we have $\left|\lambda_{1}^{m_{l}}+\cdots+\lambda_{n}^{m_{l}}\right|<1$ we know that $1-\left(\lambda_{1}^{m_{l}}+\cdots+\lambda_{n}^{m_{l}}\right)$ cannot be an integer. This is not possible.

Case 3. Suppose that $\left|\lambda_{1}\right|=1$. We will show that the only possibility is that $\lambda=1, \lambda_{2}, \ldots, \lambda_{n}$ $=0$ and we have no fixed points.

Suppose that $\left|\lambda_{1}\right|=\cdots=\left|\lambda_{k}\right|=1$ for some maximal $1 \leq k \leq n$.

Case $3 a$. First suppose that $k \geq 2$. For large $m, \operatorname{Re}\left(\lambda_{k+1}^{m}+\cdots+\lambda_{n}^{m}\right)$ is very small. Again, by Lemma 2.3, for any $\epsilon>0$ we have some sequence $m_{l} \rightarrow \infty$ such that

$$
\operatorname{Re}\left(\lambda_{1}^{m_{l}}+\cdots+\lambda_{k}^{m_{l}}\right)>k(1-\epsilon)
$$

So, since $k \geq 2$, for $m_{l}$ large enough we have $1-\lambda_{1}^{m_{l}}+\cdots+\lambda_{n}^{m_{l}}$ negative, a contradiction by Theorem 2.1 .

Case $3 b$. Suppose that $k=1$. If $\lambda_{1}$ were not real then $\overline{\lambda_{1}}$ would also be an eigenvalue, so $k$ could not be 1 . Therefore, $\lambda_{1}= \pm 1$. We may suppose that $\lambda_{i}=0$ for $1<i \leq n$ (the case where $0<\left|\lambda_{2}\right|$ is clear because as in Case 2, for large $m, L\left(f^{m}\right)$ will not be an integer). If $\lambda_{1}=1$ then $L\left(f^{m}\right)=0$ for all $m$ so there are no fixed points of $f^{m}$.

If $\lambda_{1}=-1$ then $L(f)=2$ and $L\left(f^{2}\right)=0$. By the Lefschetz Fixed Point theorem, $L(f)=$ 2 implies that $f$ has a fixed point. Therefore, $f^{2}$ has a fixed point and by Theorem 2.1, $L\left(f^{2}\right) \geq 1$ : so we have a contradiction.

Case 4. Suppose that all the eigenvalues are zero then $L\left(f^{m}\right)=1$ for all $m \in \mathbb{N}$. Therefore, $f$ has a fixed point and $\operatorname{Per}(f)=\{1\}$. This completes the proof of the theorem.

\section{Acknowledgment}

The first author was partially supported by an MCYT Grant BFM2002-04236-C02-02 and by a CIRIT Grant number 2001SGR 00173 and the second one by a Marie Curie Fellowship number HPMT-CT-2001-00247. 


\section{References}

[1] R. F. Brown, The Lefschetz Fixed Point Theorem, Scott, Foresman and Company, Illinois, 1971.

[2] C. J. Earle and R. S. Hamilton, A fixed point theorem for holomorphic mappings, Global Analysis (Proc. Sympos. Pure Math., Vol. 16, Berkeley, Calif, 1968), American Mathematical Society, Rhode Island, 1970, pp. 61-65.

[3] N. Fagella and J. Llibre, Periodic points of holomorphic maps via Lefschetz numbers, Trans. Amer. Math. Soc. 352 (2000), no. 10, 4711-4730.

[4] L. A. Harris, Fixed points of holomorphic mappings for domains in Banach spaces, Abstr. Appl. Anal. 2003 (2003), no. 5, 261-274.

[5] J. X. Hou, Periodic points of analytical dynamical systems on hyperbolic Riemann surfaces, Acta Sci. Natur. Univ. Sunyatseni 1988 (1988), no. 1, 28-33.

[6] J. Milnor, Notes on dynamical systems, www.math.sunysb.edu/ jack/DYNOTES/.

[7] Dynamics in One Complex Variable, 2nd ed., Friedr. Vieweg \& Sohn, Braunschweig, 1999.

[8] G. Pick, Über einer eigenschaft der konformen abbildung kreisförmiger berieche, Math. Ann. 77 (1916), 1-6 (German).

[9] J. W. Vick, Homology Theory, 2nd ed., Graduate Texts in Mathematics, vol. 145, SpringerVerlag, New York, 1994.

Jaume Llibre: Departament de Matemàtiques, Universitat Autònoma de Barcelona, 08193 Bellaterra, Barcelona, Spain

E-mail address: jllibre@mat.uab.es

Michael Todd: Department of Mathematics and Statistics, University of Surrey, Surrey, Guildford GU2 7XH, UK

E-mail address: m.todd@surrey.ac.uk 


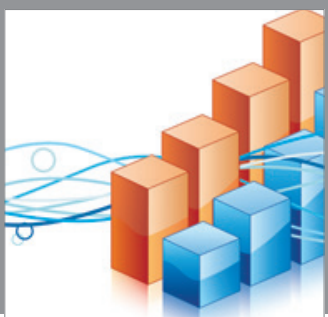

Advances in

Operations Research

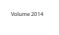

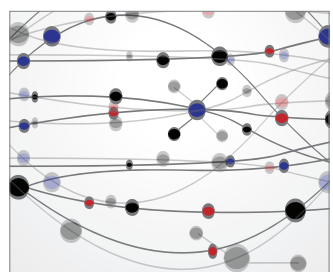

\section{The Scientific} World Journal
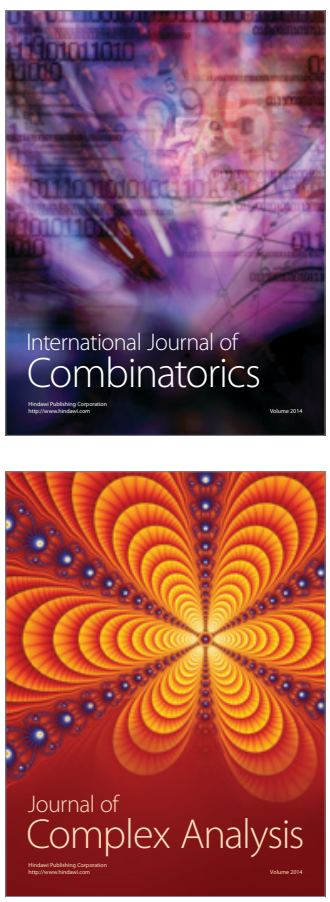

International Journal of

Mathematics and

Mathematical

Sciences
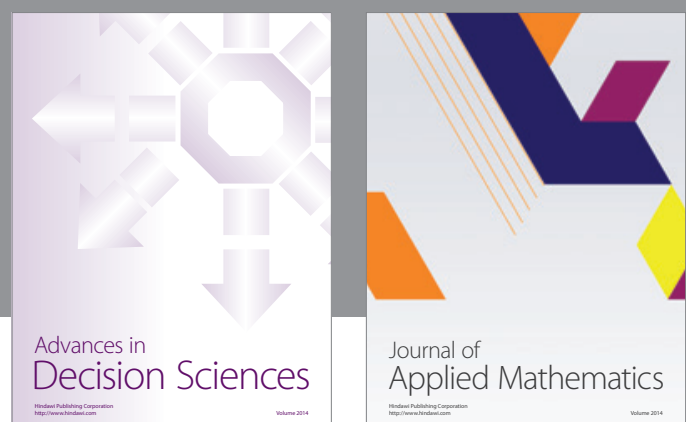

Journal of

Applied Mathematics
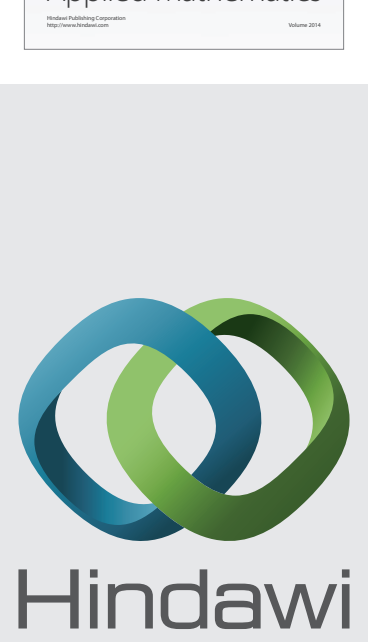

Submit your manuscripts at http://www.hindawi.com
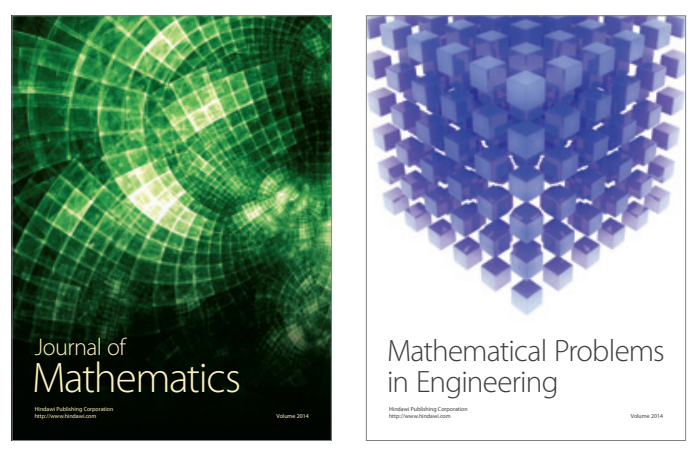

Mathematical Problems in Engineering
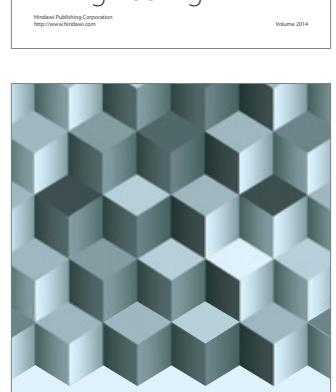

Journal of

Function Spaces
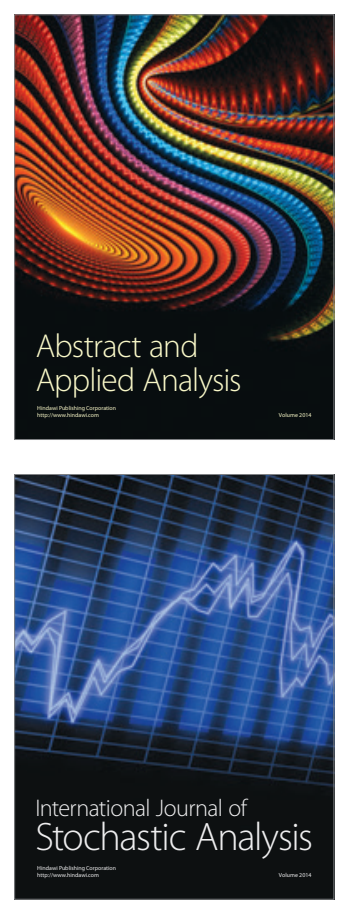

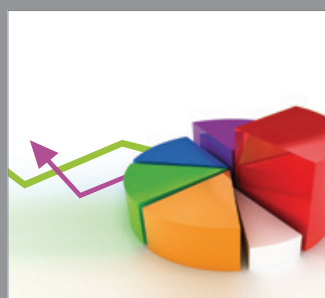

ournal of

Probability and Statistics

Promensencen
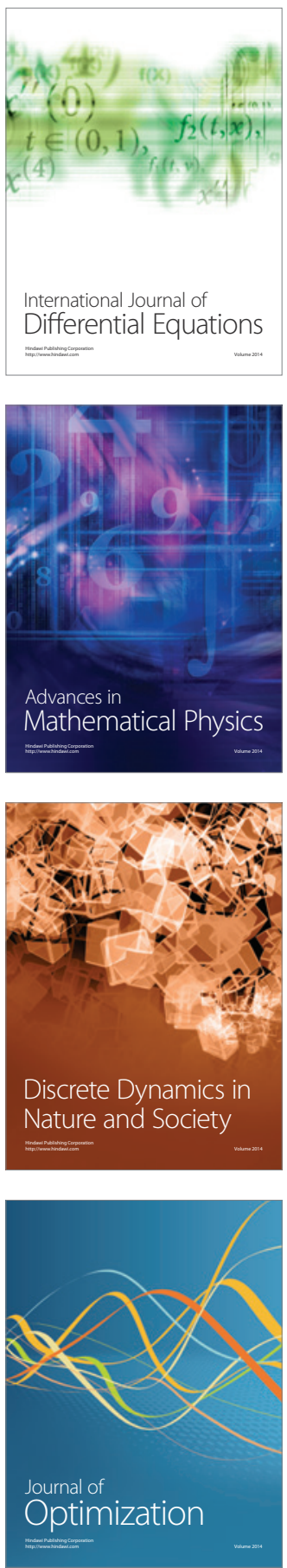